\section{Neue Echinodera aus Griechenland - Beitrag zur integrativen Taxonomie (Coleoptera: Curculionidae: Cryptorhynchinae)}

\author{
Mit 13 Figuren
}

Peter E. StüBen ${ }^{1}$ und Katja Kramp ${ }^{2}$

1 Curculio Institute, Hauweg 62, 41066 Mönchengladbach, Germany. - P.Stueben@t-online.de

2 Senckenberg Deutsches Entomologisches Institut, Eberswalder Straße 90, 15374 Müncheberg, Germany.

- Katja.Kramp@senckenberg.de

Published on 2019-12-23

DOI:10.21248/contrib.entomol.69.2.319-330

\section{Abstract}

Two new species, Echinodera magnesia and E. arcadia, and the subspecies E. brachati peloponnensis are described by the first author from Greece (Peloponnese and Pilion Mountains) and are distinguished from the related species. They are incorporated into the current key of the Mediterranean species of the genus. In addition to morphological characters, first molecular analyses (barcoding) are presented, and the new taxa are integrated into a dendrogram of the Southeast European Echinodera species (Maximum-Likelihood tree; CO1, 658 bp).

\section{Nomenclatural acts}

Echinodera magnesia STÜBEN spec. nov. - urn:lsid:zoobank.org:act:90FF8B7B-7DC2-4214-83E9-075D004C34CF Echinodera arcadia STÜBEN spec. nov. - urn:lsid:zoobank.org:act:6E89A7D2-4C51-4FD2-A847-5E92719EC9E7 Echinodera brachati peloponnensis STÜBEN ssp. nov. - urn:lsid:zoobank.org:act:76479EE7-91A7-4F57-BC0B-AFD9C1 D0F2A1

\section{Key words}

Coleoptera, Curculionidae, Cryptorhynchinae, Echinodera, new species, morphology, molecular analysis, barcoding, integrative taxonomy, distribution map, Greece

\section{Zusammenfassung}

Zwei neue Arten, Echinodera magnesia und E. arcadia, sowie die Unterart E. brachati peloponnensis werden vom Erstautor beschrieben (Peloponnes und Pilion Gebirge) und von den verwandten Arten morphologisch unterschieden. Die neuen Taxa werden in den bekannten Schlüssel der mediterranen Echinodera-Arten eingebunden. Über die morphologischen Merkmale hinausgehend werden erste molekulare Analysen (Barcodes) vorgestellt, und die neuen Arten in einem Dendrogramm - Maximum-Likelihood-Baum (CO1, 658 bp) - der südosteuropäischen Echinodera berücksichtigt. 


\section{Schlüsselwörter}

Coleoptera, Curculionidae, Cryptorhynchinae, Echinodera, neue Arten, Morphologie, molekulare Analyse, Barcodes, integrative Taxonomie, Verbreitungskarten, Griechenland

\section{Einleitung}

Zwei längere Sammelreisen führten den Erstautor in den letzten beiden Jahren in den Norden Griechenlands (Mount Olympus, Pilion-Gebirge, 2017) und auf die Peloponnes (2018). Die CryptorhynchinaeFauna Griechenlands ist bei weitem nicht so reich an Arten wie etwa der Südwesten Europas oder der Nordwesten Afrikas, ganz zu schweigen von den Makaronesischen Inseln [STÜBEN 2018]. Dennoch wurden in den letzten zwei Jahrzenten hier mehrere EchinoderaArten entdeckt und beschrieben: Echinodera behnei STÜвеN, 1998 (Gr.: Makedonien), E. ingowolfi STÜBEN, 1998 (Astakos), E. brachati Wolf, 2002 (Peloponnes), E. (Ruteria) montissacris (BeHne, 2002) (Chalkidike), E. athosensis BeHne, 2005 (Mt. Athos), Echinodera (Ruteria) zerchei (BEHNe, 2002) (Pangeó Mts.), E. corcyrensis STÜBEN, 2008 (Korfu) und E. (Ruteria) soumasi Germann, Wolf \& Schütte 2015 (Peloponnes); siehe auch [Fig. 12].

Die bisherige Annahme, dass das disjunkte Verbreitungsgebiet der vom Erstautor bereits 1998 beschriebenen Art Echinodera behnei (Locus typicus: Pangéo Mts.) ausgehend von den Lakatischka Rila Mts. über die Rhodopen Bulgariens bis auf die griechische Halbinsel Chalkidike und - davon getrennt - die Gebirge östlich von Larissa reiche [STÜBEN 2018], muss korrigiert werden. Tatsächlich gehören die Exemplare aus den Gebirgen des Pilion zu der neuen Art Echinodera magnesia [Fig. 1], die in der Monographie über die westpaläarktischen Cryptorhynchinae noch unter dem (alten) Namen Echinodera behnei abgebildet wurde [STüBEN 2018: 282]. Beide Arten trennen nicht so sehr morphologische Merkmale (siehe Erstbeschreibung), die bei den Echinodera tatsächlich nur sehr begrenzte und marginale Einblicke in die Evolution und Phylogenie der Arten vermitteln, sondern vor allem auffällig große molekulare Distanzen (etwa beim mitochondrialen CO1-Barcoding-Gen). Auf eine ausführliche Beschreibung der neuen Arten, die nur zum wiederholten Male die wenigen immer gleichen morphologischen Merkmale aller Echinodera-Arten protokolliert, wird diesmal zugunsten aussagekräftigerer Differentialdiagnosen verzichtet. Außerdem werden für die neuen und verwandten Arten ein molekulares Dendrogramm [Fig. 13] und eine Distanzmatrix (siehe Anhang 1) vorgestellt sowie Barcoding-Sequenzen in GenBank hinterlegt, die zukünftig eine eindeutige $(\mathrm{Re})$ identifikation der neuen Taxa erlauben.

\section{Echinodera magnesia STüBEN spec. nov.} urn:Isid:zoobank.org:act:90FF8B7B-7DC2-4214-83E9-075D004C34CF (Fig. 1, 2, 4, 13)

Typenmaterial: Holotyp: $10^{\text {* }}$, "Greece, (Magnesia), Pilion Mt., 2 km SW Zagora <N39 25'33"E235'25">, 500 m, Quercus, 20.7.1998, leg. Stüben (65)“, coll. Senckenberg, Deutsches Entomologisches Institut (SDEI). //

Paratypen: $4 \sigma^{-} \sigma^{-1}, 1$, Angaben wie bei Holotyp, coll. Stüben; $2 \sigma^{\star} \sigma^{\star}, 2$ ㅇ 우, „Greece, Pilion Mt. (Magnesia): $6.5 \mathrm{~km}$ SW Agios Dimitrios, 39²3'18"N 234'50"E, 1293 m, Fagus, 6.7.2017, leg. Stüben (14)“, coll. Stüben; $10^{\star}, 1$ ㅇ, „Greece, Pilion Mt. (Magnesia): 2 km W Chania, $39^{\circ} 23^{\prime} 59^{\prime \prime N} 23^{\circ} 2^{\prime} 16^{\prime E}, 1461 \mathrm{~m}$, Fagus, 6.7.2017, leg. Stüben (15)“, coll. Stüben; 1 , „Greece, (Magnesia), Pilion Mt., N Lampinou, 39²1'19"N 2311'53"E, 353 m, Platanus, 7.7.2017, leg. Stüben (17)“, coll. Stüben.

DNA-Typen (CO1) (= Paratypen): 1 Ex, „Greece, (Magne-

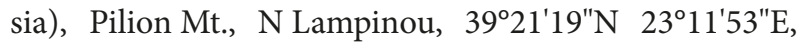
353 m, Platanus, 7.7.2017, leg. Stüben (17)", coll. Stüben, Sammler-Nr.: 2983-PST, GenBank: MK347561 // 2 Ex.: „Greece, Pilion Mt. (Magnesia): 2 km W Chania, $39^{\circ} 23^{\prime} 59^{\prime \prime} \mathrm{N} 23^{\circ} 2^{\prime} 16^{\prime E}, 1461 \mathrm{~m}$, Fagus, 6.7.2017, leg. Stüben (15)“, coll. Stüben, Sammler-Nr.: 3094-PST, GenBank: MK347644 // 1 Ex.: „Greece, Pilion Mt. (Magnesia): $6.5 \mathrm{~km}$ SW Agios Dimitrios, 39²3'18"N 23ํ'50"E, 1293 m, Fagus, 6.7.2017, leg. Stüben (14)“, coll. Stüben, Sammler-Nr.: 2977-PST, GenBank: MK347560. Weiteres Material vom Pilion-Gebirge befindet sich in meiner Cryptorhynchinae-Sammlung des Deutschen Entomologischen Instituts, Senckenberg (Müncheberg) unter dem (alten) Artnamen „E. behnei“.

Beschreibung und Differentialdiagnose: Größe: 2,4-3,4 mm (ohne Rüssel).

Die im Durchschnitt etwas größere Art entspricht in den äußeren Merkmalen im Wesentlichen der aus Nordgriechenland und Bulgarien beschriebenen Art Echinodera behnei [STÜBEN 1998]. Jedoch sind die Elytren langoval und weniger auffällig eiförmig zum Apex hin verrundet. Die senkrecht aufstehenden, hellbraunen, annähernd parallelseitigen Borsten(nadeln) sind ca. $4 \mathrm{x}$ länger als breit, während die zur Spitze hin keulenförmigen, etwas kürzeren Borsten von E. behnei zur Elytrenspitze hin etwas geneigt und in sich leicht gekrümmt sind. Die neue Art besitzt breite Elytrenintervalle, die auf der Elytrenscheibe so breit oder breiter als die Punktstreifen sind; während sie - ähnlich wie E. behnei - auf den Elytrenflanken stark vergrößerte, grubenartige Punkte besitzt, die auf die Elytrenintervalle übergreifen. 


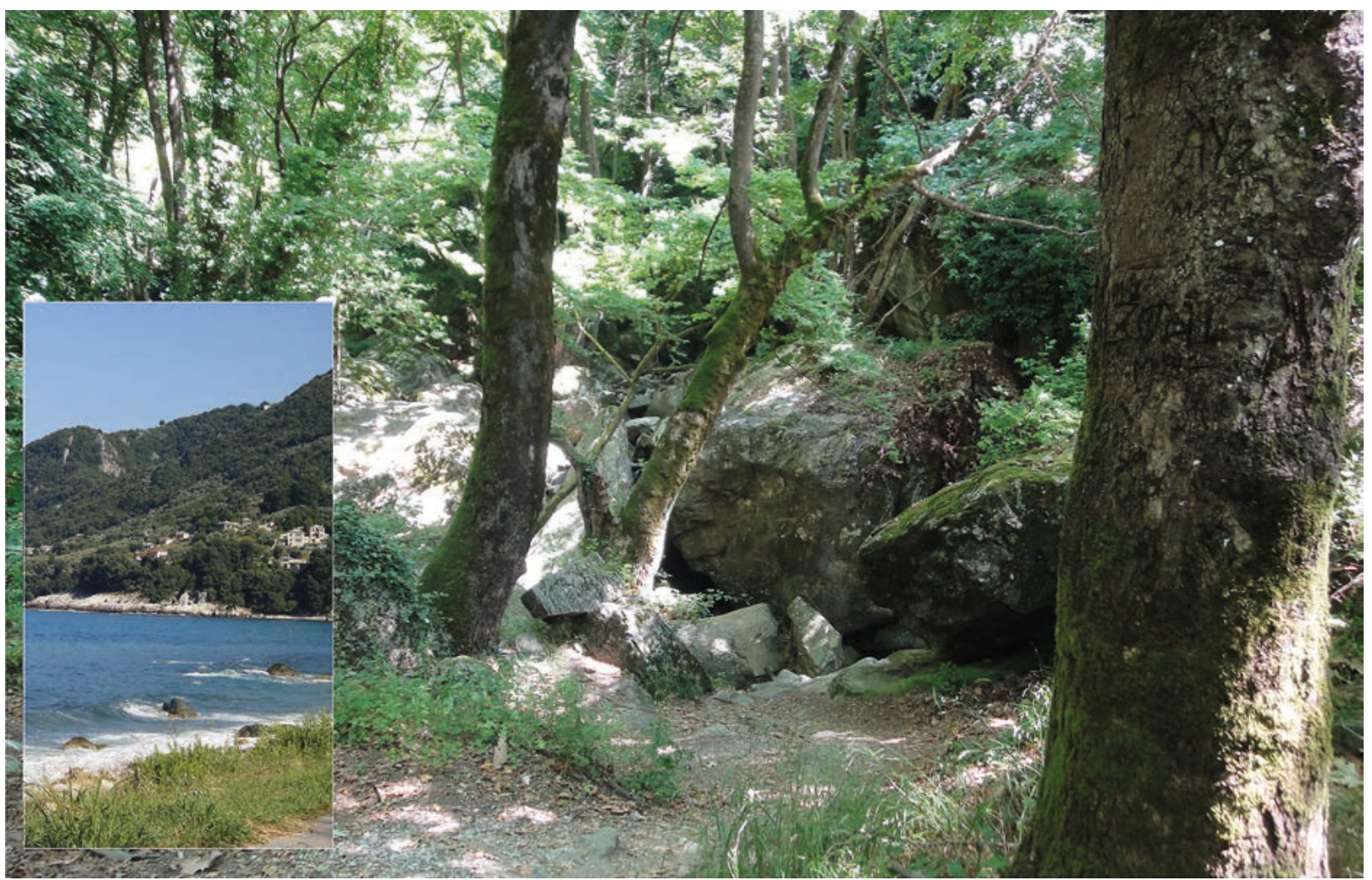

Fig. 1: In den dichten Wäldern des Pilion-Gebirges im Norden Griechenlands fand sich die neue Art Echinodera magnesia praktisch auf allen Höhenstufen vom Meer bis in die Fagus-Wälder über 1400 m. Aufn. Stüben, 7.7.2017.

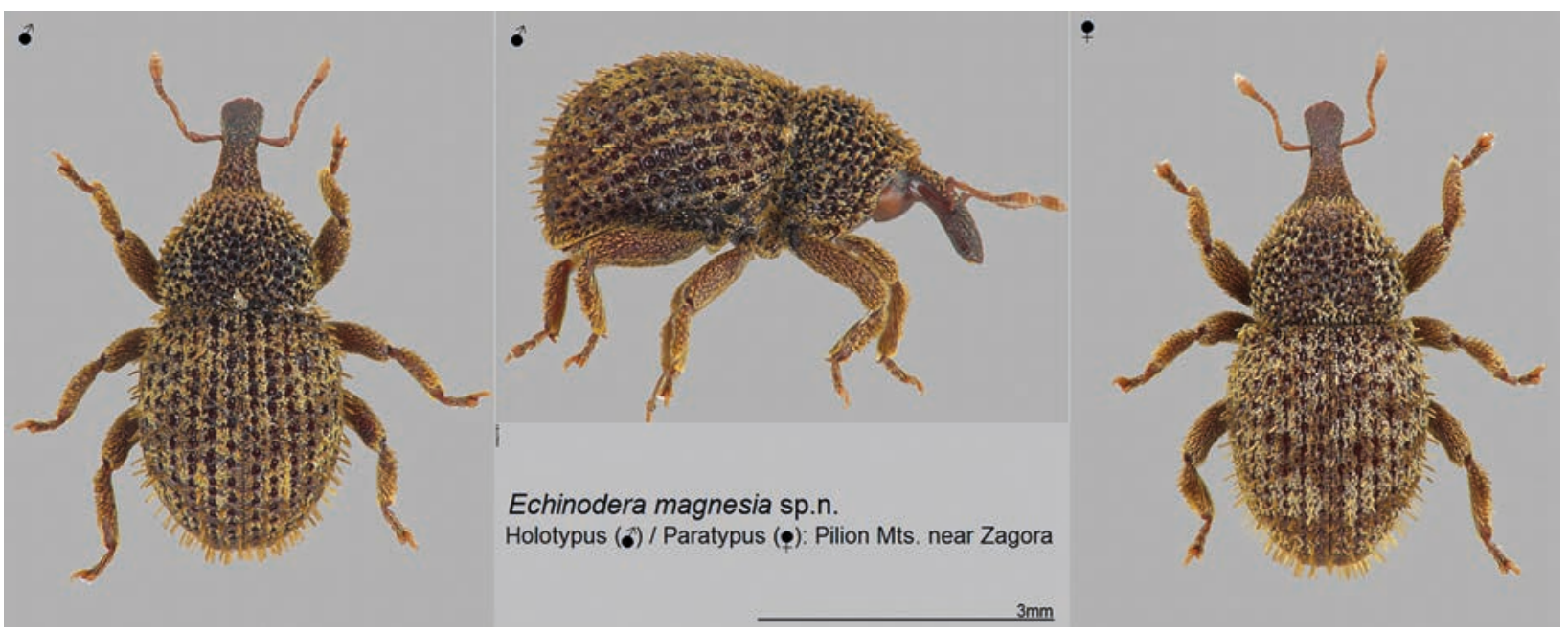

Fig. 2: Echinodera magnesia spec. nov.

E. magnesia ist auf den Intervallen dicht beschuppt; eine deutliche Binden- oder Fleckenzeichnung ist auf den mit beigefarbenen Schuppen durchmischten braunen Elytren nicht zu erkennen.

Das Pronotum ist breiter als lang und wie bei E. behnei hinter der Mitte am breitesten, stark nach vorne und hinten verrundet. Die Punktur ist grob und tief; jeder Grube entspringt eine steil abstehende helle Borste, die jedoch deutlich kürzer als auf den Elytren ist. Fühler rotbraun; Rostrum in beiden Geschlechtern grob und zwischen den Fühlereinlenkungen leicht längsrunzelig punktiert.
Aedoeagus in lateraler Ansicht zur Spitze hin etwas weniger gekrümmt als bei E. behnei: [Fig. 4, 5].

Einordnung der neuen Art in den Schlüssel der mediterranen, südeuropäischen Echinodera-Arten (dazu ziehe heran: STÜBEN 2018: 278):

[1] Haken am Ende der Hinterschiene bei den Männchen gerade / [2] Borsten senkrecht abstehend und mindestens $2 \mathrm{x}$ länger als breit / [3'] Borsten $2 \mathrm{x}-5 \mathrm{x}$ länger als breit (nicht $8 \mathrm{x}-10 \mathrm{x}$ wie bei E. capiomonti) / [4] Streifen auf den Elytrenflanken mit einer tiefen Punktur (wie bei E. behnei) / [5] Borsten $4 \mathrm{x}$ länger als breit, Elytren nicht eiförmig (wie bei E. behnei, Borsten bei dieser Art deutlich kürzer): [Fig. 2, 3]. 


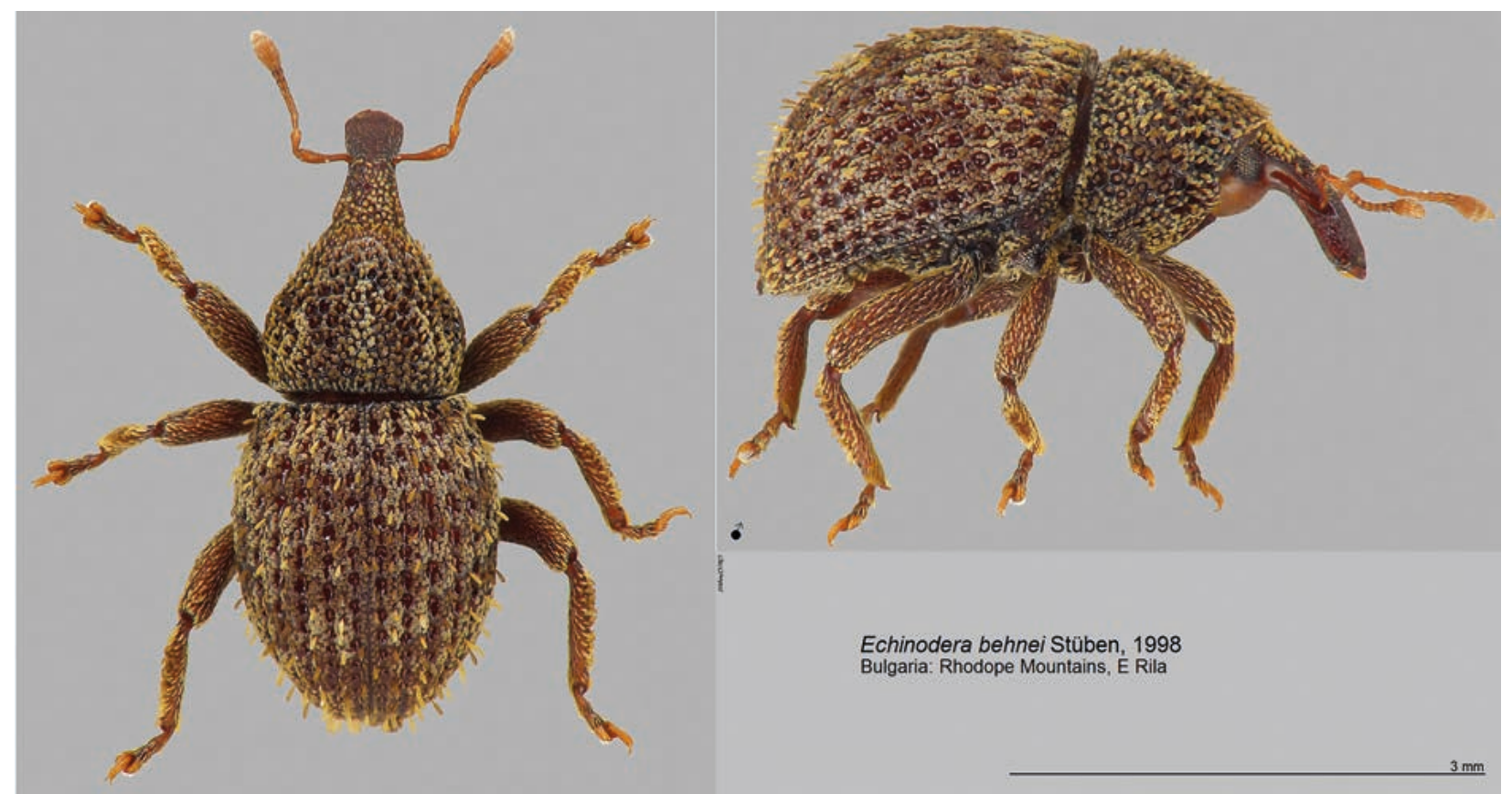

Fig. 3: Echinodera behnei StüвEN, 1998.
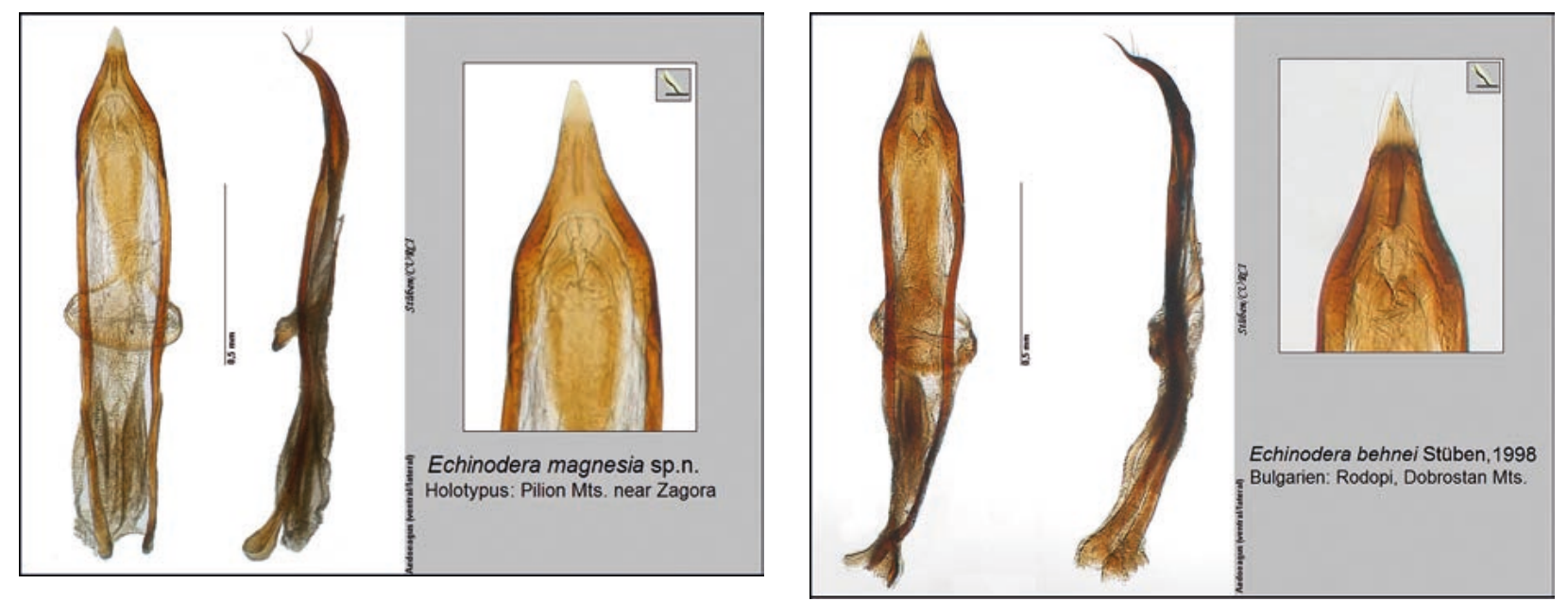

Fig. 4, 5: Aedoeagi ventral / lateral: Echinodera magnesia im Vergleich mit Echinodera behnei.

Die Art ist also im bekannten Echinodera-Schlüssel zwischen E. behnei und E. orientalis zu platzieren. Von den nachfolgenden Arten mit einem geraden Hinterschienen-Enddorn bei den Männchen hat nur noch E. romanboroveci (von Montenegro) sehr lange Elytrenborsten, doch die Elytren sind kurzoval bis rund (und nicht wie bei der neuen Art langoval).

Zur molekularen Einordnung der Art im EchinoderaDendrogramm siehe Figur 13.

Ökologie: Die Art kann aus der Laub- und Ästchenstreu (vor allem von Fagus) auf fast allen Höhenstufen des Pilion-Gebirges gesiebt werden.

Derivatio nominis: Die Art wurde nach ihrem Fundort

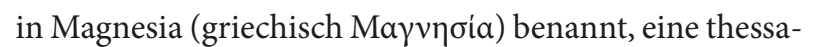
lische Landschaft im antiken Griechenland, heute Teil des Regionalbezirkes Magnisia.

\section{Echinodera arcadia StüBEN spec. nov.}

urn:Isid:zoobank.org:act:6E89A7D2-4C51-4FD2-A847-5E92719EC9E7

[Fig. 6, 7, 9, 13]

Typenmaterial: Holotyp: $1 \sigma^{*}$, "Greece, Arkadia, N Tripoli: Moni Epano Chrepas, Quercus, gesiebt, 24.8.2018, 37³2'32"N 22¹9'37"E, 1133 m, leg. Stüben (22)“, coll. Senckenberg, Deutsches Entomologisches Institut (SDEI). // Paratypen: $1 \sigma^{\star}$, Angaben wie bei Holotyp, coll. Stüben.

DNA-Type (CO1) (= Holotyp): 1 Ex.: Angaben wie bei Holotyp, coll. Stüben, Sammler-Nr.: 3172-PST, GenBank: MK347702.

Beschreibung: Größe: 2,6-3,0 mm (ohne Rüssel). Elytren: langoval, nach vorne nur wenig, zur Spitze hin breit verrundet: $1,19 x-1,23 x$ länger als breit; breiteste Stelle am Ende des 1. basalen Drittels; die Elytrenschei- 


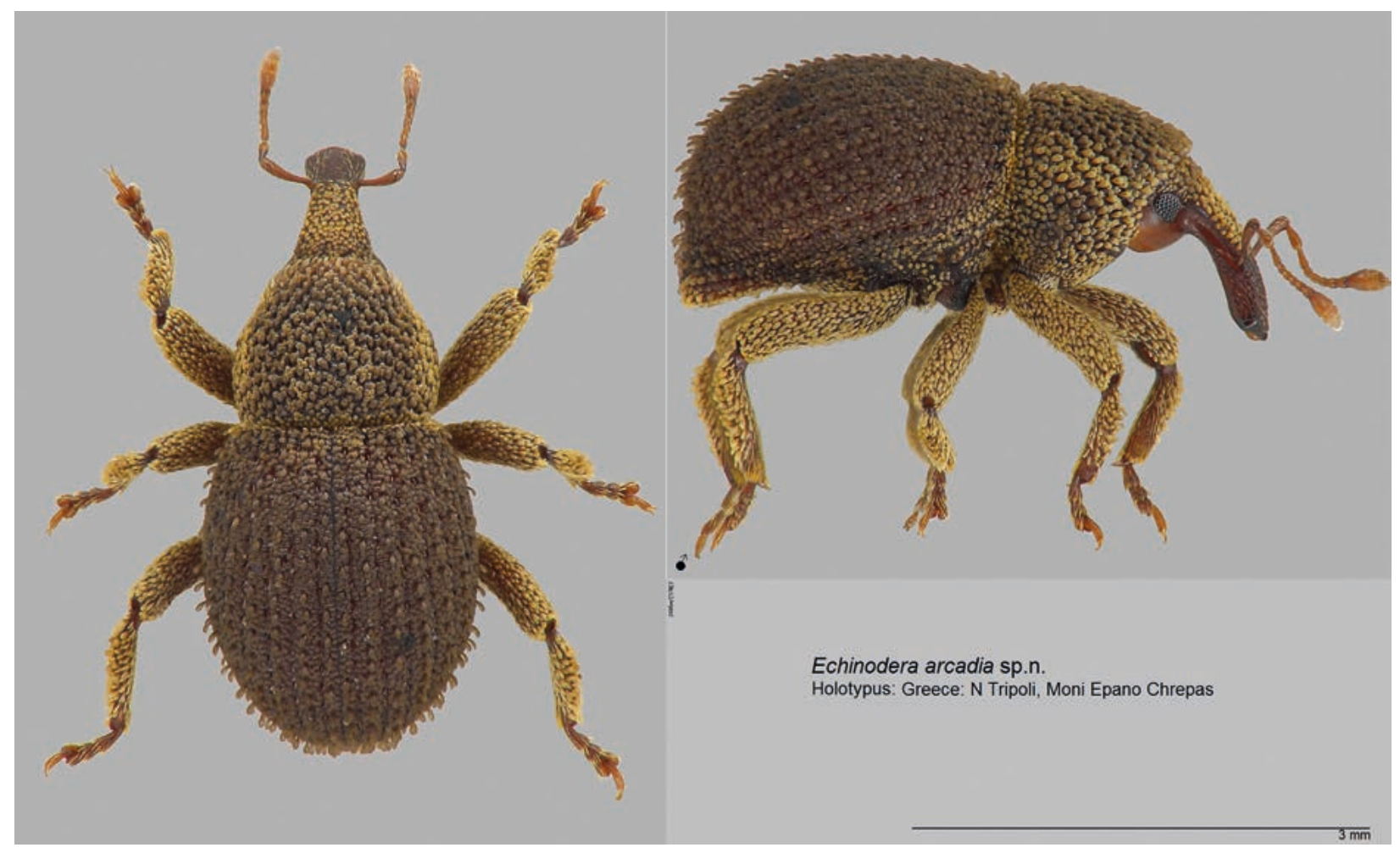

Fig. 6: Echinodera arcadia spec. nov.
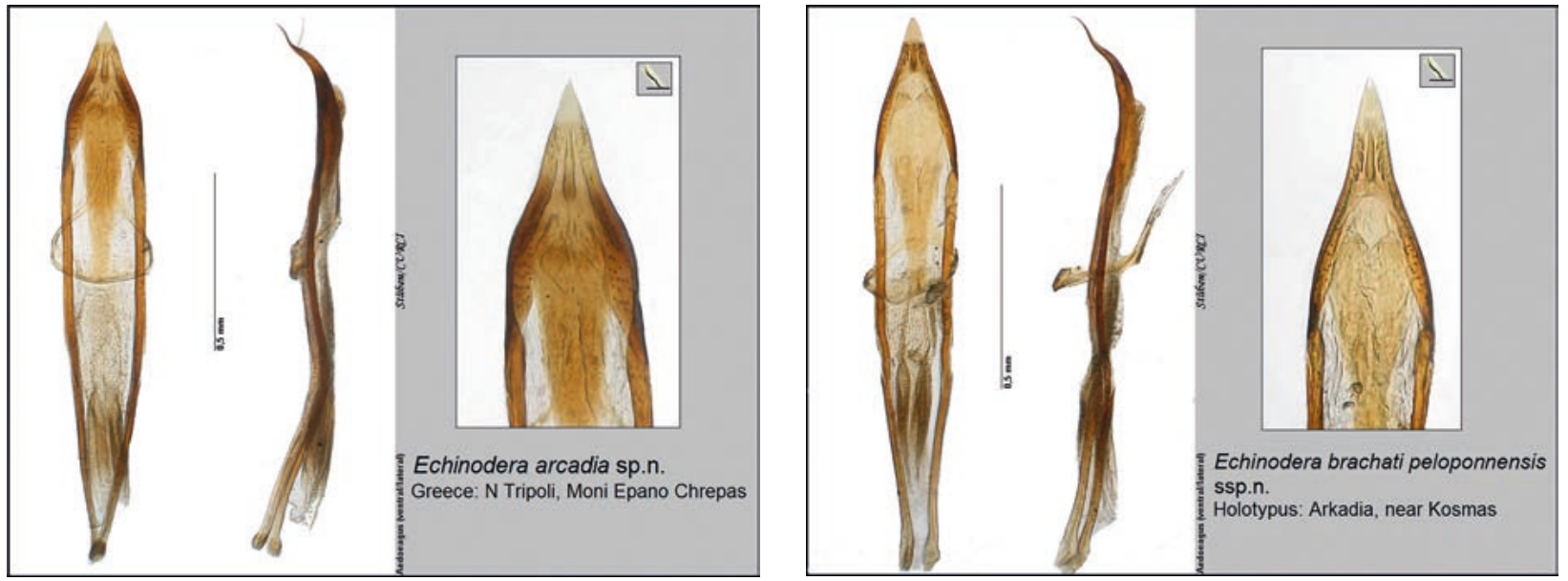

Fig. 7, 8: Aedoeagi (ventral / lateral) der beiden neuen Arten von der Peloponnes-Halbinsel.

tellinie, die das Pronotum etwas überragt, bei lateraler Betrachtung zunächst hinter der Basis flach, sie bildet erst weit hinter der Mitte einen Kreisbogen, der zur Spitze hin senkrecht (bzw. sogar etwas einwärts gebogen) abfällt; Elytrenbasis (auffallend) gerade. Das Integument besteht vollständig aus dunkelbraunen Schuppen ohne irgendwelche Aufhellungen, Binden- oder Fleckenzeichnungen (Alleinstellungsmerkmal unter den Echinodera-Arten!). Die wie an einer Perlenschnur aufgereihten kurzen, breiten, höchstens 1,3x längeren als breiten Elytrenborsten abstehend, nur leicht geneigt und mit breiten Zwischenräumen; die etwas gewölbten Intervalle um ein Vielfaches breiter als die schmalen (rinnenartigen) Punktstreifen.

Pronotum: Untersetzt, $1,23 \mathrm{x}-1,27 \mathrm{x}$ breiter als lang; breiteste Stelle am Ende des 1. basalen Drittels; dort bauchig verrundet, zum Vorderrand hin gradlinig enger werdend; ohne eine seitliche Depression. Pronotumscheibe nur geringfügig vor der Basis gewölbt, zum Vorderrand hin flach auslaufend. Integument besteht ebenfalls aus dunkelbraunen Schuppen, die zu den Seiten hin in eine beigefarbene Zeichnung übergeht (ohne markante Fleckenbildung); jedem feinen Punktgrübchen entspringt eine kurze, breite, spatelförmig abgeflachte und nach vorn gerichtete Schuppe.

Aedoeagus: Medianlobus bei lateraler Betrachtung zur Spitze hin stark gebogen [Fig. 7].

Differentialdiagnose und Diskussion: Die neue Art ist leicht von allen anderen südeuropäischen Echinodera-Arten aufgrund ihres einfarbig dunkelbraunen Schuppenkleides zu unterscheiden (Alleinstellungsmerk- 


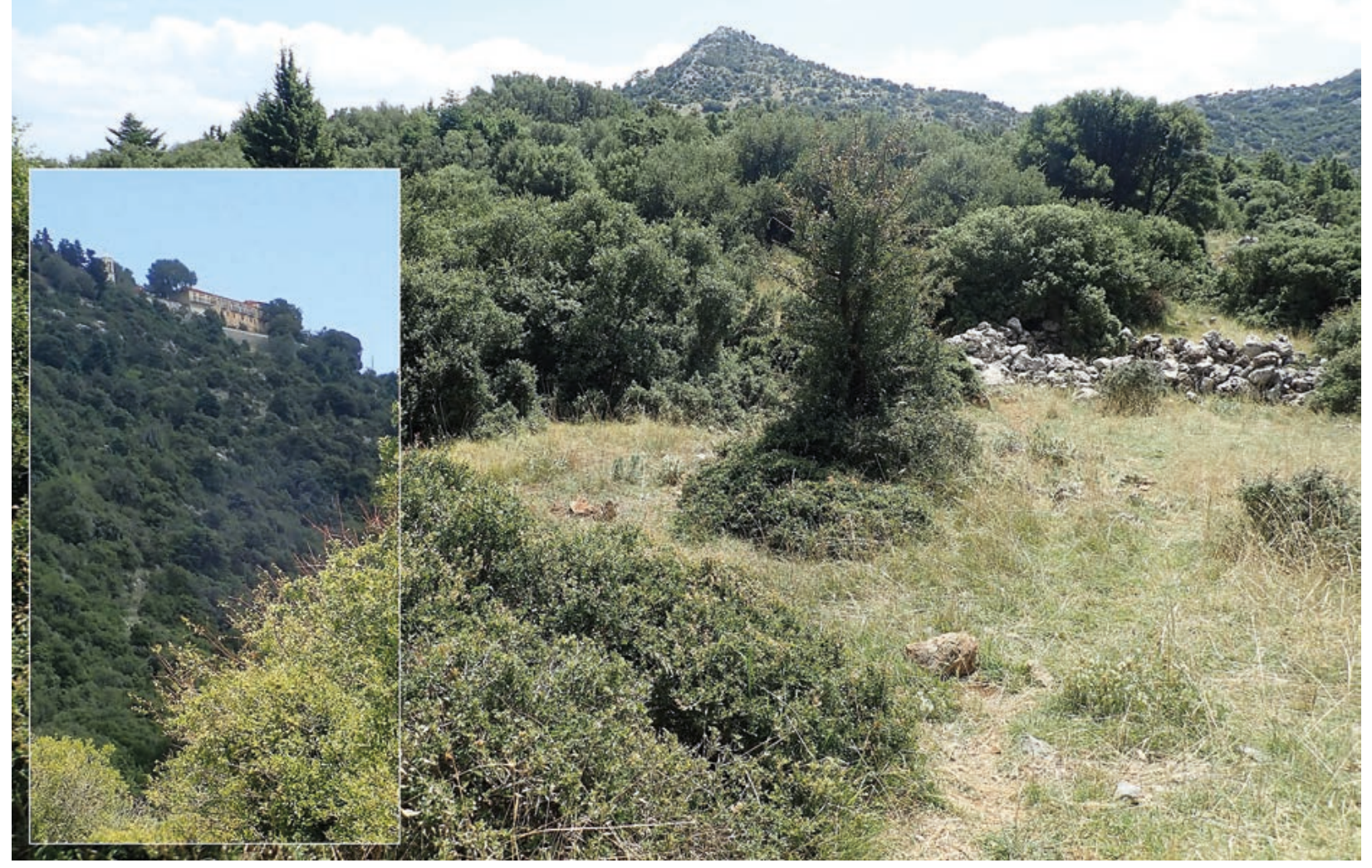

Fig. 9: Unmittelbar unterhalb des Klosters von Epano Chrepas nördlich von Tripoli (Peloponnes) wurde die neue Art E. arcadia aus der Laub- und Ästchenstreu der dort dichten - leider von Ziegen zerfurchten - Quercus-Sträucher in einer Höhe von $1100 \mathrm{~m}$ gesiebt. Aufn. Stüben, 24.8.2018.

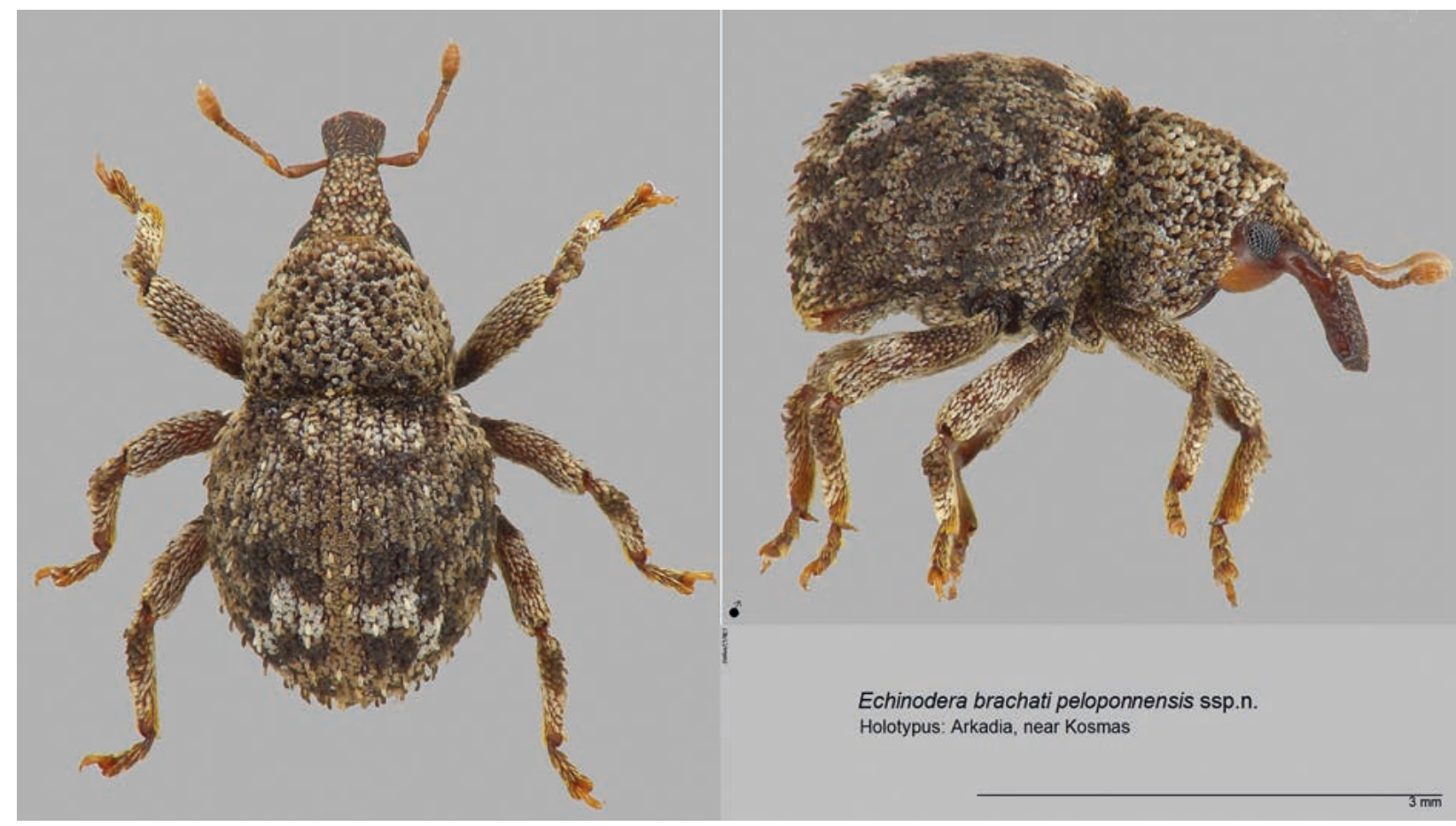

Fig. 10: Echinodera brachati peloponnensis ssp. nov.

mal) und gehört zu den Arten mit einem geraden Hinterschienen-Enddorn bei den Männchen [Fig. 6]. E. arcadia ordnet sich wie folgt in den bekannten Schlüssel der mediterranen, südeuropäischen Echinodera-Arten ein (dazu: SтÜвеN 2018: 278):
[1] Haken am Ende der Hinterschiene bei den Männchen gerade / [2'] Borsten kurz, höchstens 1,3x länger als breit / [13'] Elytrenborsten angehoben / [14'] Kleine Arten, max. 3,0 mm / [15] die gestreckten Elytren und das Pronotum einfarbig dunkelbraun (nicht braun-weiß- 


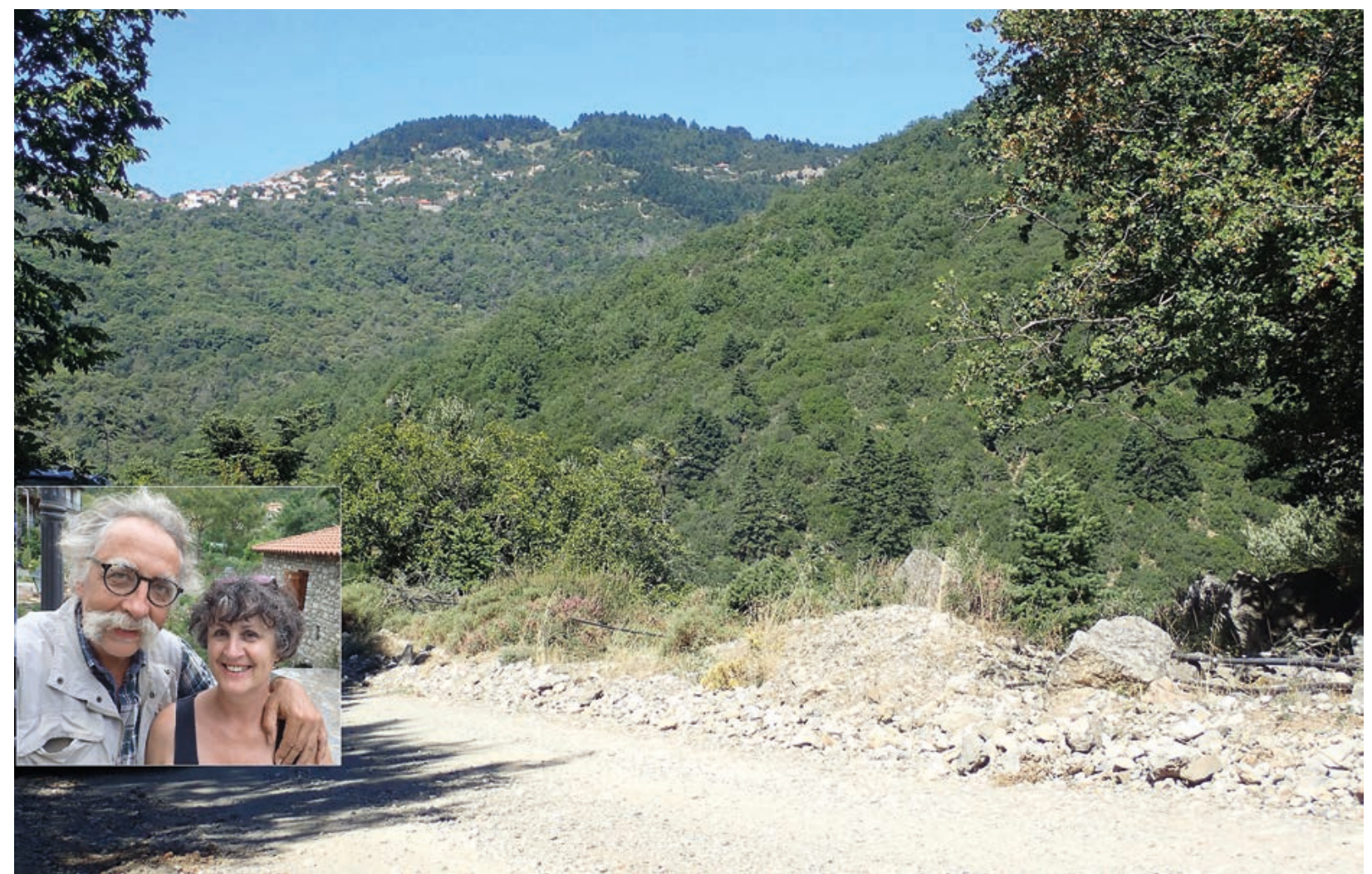

Fig. 11: Tatkräftige Unterstützung: Regine und Peter Stüben siebten die neue Unterart E. brachati peloponnensis unterhalb von Kosmas im Parnon-Gebirge unter Astragalus-Polstern im Schatten von Quercus-Sträuchern im August 2018. Aufn. Stüben \& unbekannter Hirte, 11.08.2018.

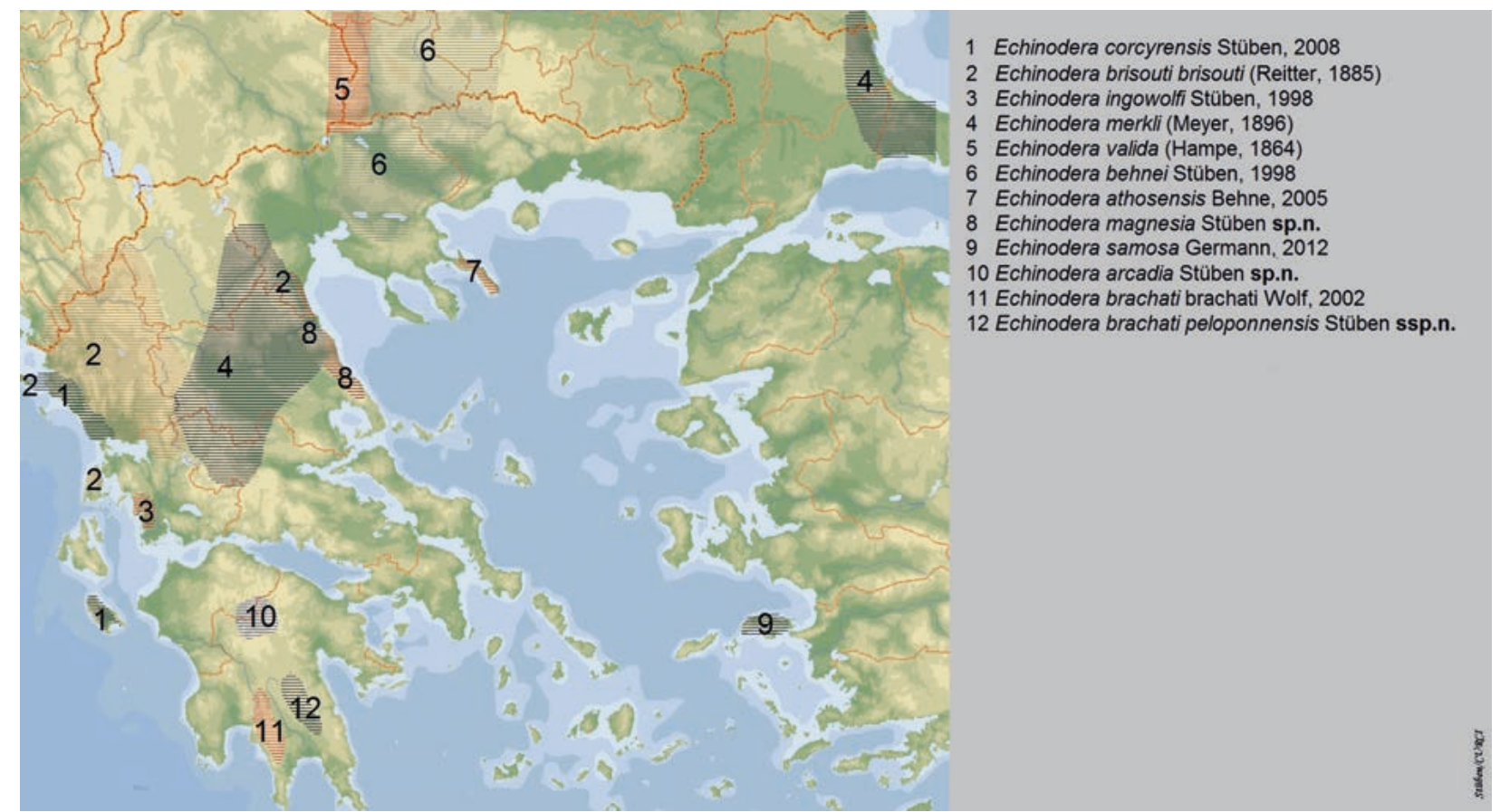

Fig. 12: Bisher bekannte Hauptverbreitungsgebiete der Echinodera-Arten (ohne die Arten des Subgenus Ruteria) auf dem griechischen Festland und der Peloponnes.

beige durchmischt wie bei E. variegata von Sizilien oder E. corcyrensis von Korfu).

Die Art ist also im Echinodera-Schlüssel vor den bekannten Arten E. variegata von Sizilien und E. corcyrensis von Korfu zu platzieren. Sie sieht - blendet man die gattungs- spezifischen Merkmale einmal aus (z. B. schmale Augen, fehlende Innensackstruktur des Aedoeagus) - vom Habitus und der monochrom braunen Farbzeichnung her den Arten um Acalles sierrae von der Iberischen Halbinsel zum Verwechseln ähnlich. 
Zur molekularen Einordnung der Art im EchinoderaDendrogramm siehe weiter unten: [Fig. 13].

Ökologie: Der Erstautor siebte die Art nördlich von Tripoli (Peloponnes) unmittelbar unterhalb des Klosters von Epano Chrepas aus der Laub- und Ästchenstreu der dort dichten - leider von Ziegen zerfurchten - QuercusSträucher in einer Höhe von $1100 \mathrm{~m}$.

Derivatio nominis: Die Art wurde benannt nach ihrem Fundort im Zentrum der Peloponnes, der Landschaft

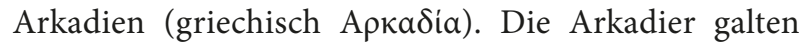
schon im Altertum als raues Hirtenvolk. Und Ziegenherden sind es noch heute, die die erodierende Landschaft prägen und das Sammeln zwischen den sie begleitenden (Wolfs-)Hunden oft zu einem Abenteuer mit ungewissem Ausgang werden lassen; mit eine Erklärung dafür, warum unter diesen Umständen von der neuen Echinodera-Art nur 3 Exemplare vom Erstautor gesiebt werden konnten.
Echinodera brachati peloponnensis STÜBEN Ssp. nov. urn:Isid:zoobank.org:act:76479EE7-91A7-4F57-BCOB-AFD9C1 DOF2A1 [Fig. 8, 10, 11, 13]

Typenmaterial: Holotyp: $1 \sigma^{*}$, "Greece, Arkadia, E Kosmas,

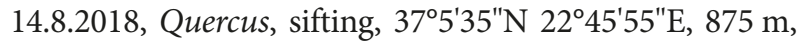
leg. Stüben (8) “, coll. Senckenberg, Deutsches Entomologisches Institut (SDEI). //

Paratypen: 1 ㅇ, „Greece, Arkadia, Kosmas, 9.8.2018, Astragalus, sifting, $37^{\circ} 5^{\prime} 45^{\prime \prime} \mathrm{N} \quad 22^{\circ} 44^{\prime} 28^{\prime \prime} \mathrm{E}, 1151 \mathrm{~m}$,

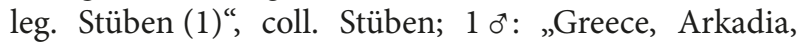
N Kosmas: near Moni Elonis, 11.8.2018, Quercus, sifting, $37^{\circ} 8^{\prime} 42^{\prime N} 22^{\circ} 45^{\prime} 3^{\prime \prime E}, 427$ m, leg. Stüben (3)”, coll. Stüben. DNA-Typen (CO1) (= Paratypen): 1 Ex.: „Greece, Arkadia, Kosmas, 9.8.2018, Astragalus, sifting, $37^{\circ} 5^{\prime} 45^{\prime \prime} \mathrm{N}$ $22^{\circ} 44^{\prime} 28^{\prime E}$, 1151 m, leg. Stüben (1)“, coll. Stüben, Sammler-Nr.: 3163-PST, GenBank: MK347695 // 1 Ex.: „Greece, Arkadia, N Kosmas: near Moni Elonis, 11.8.2018, Quercus,

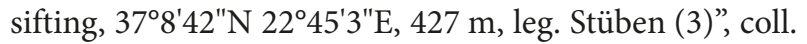
Stüben, Sammler-Nr.: 3166-PST, GenBank: MK347697.

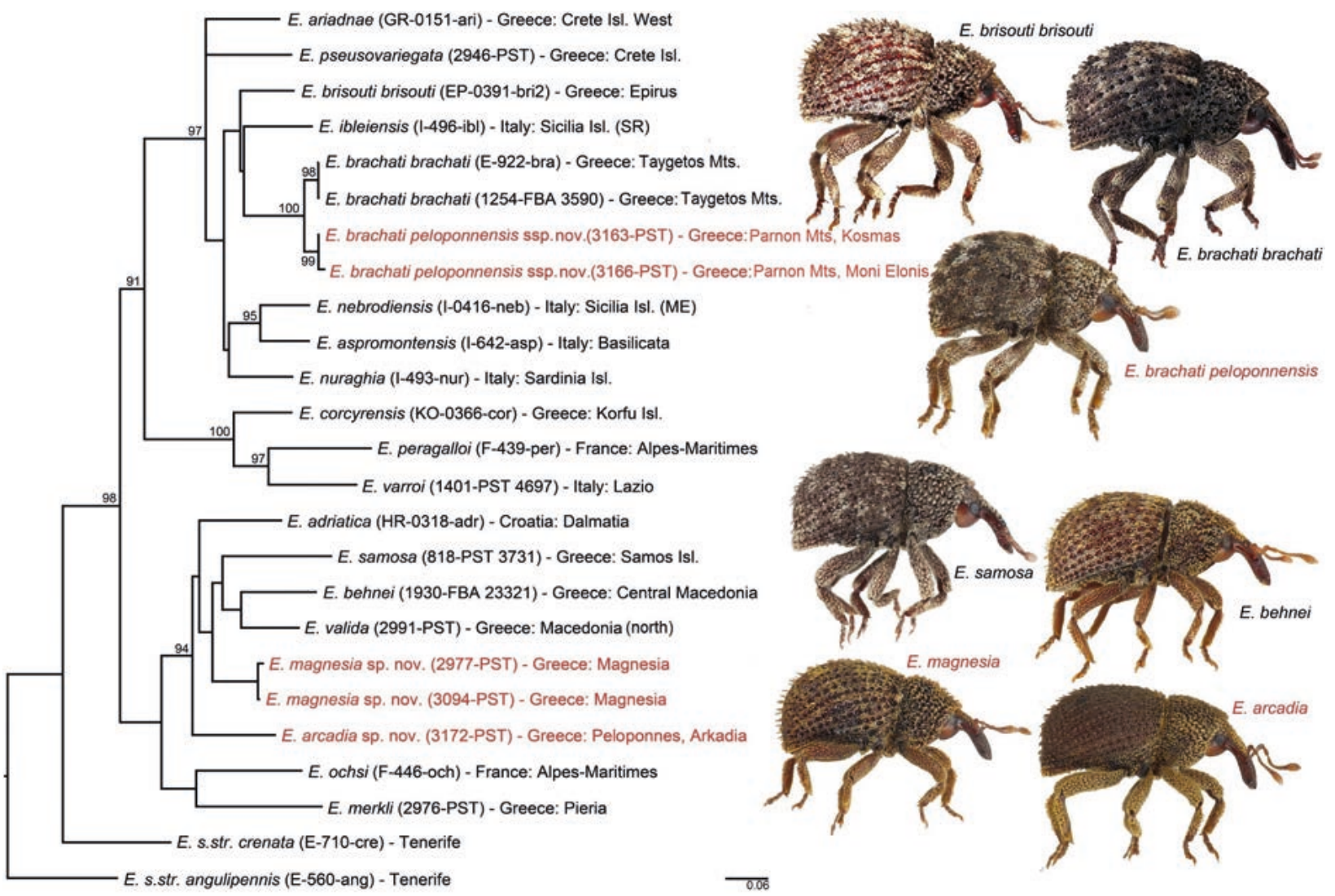

Fig. 13: Maximum-Likelihood-Baum (CO1, 658 bp) zur verwandtschaftlichen Einordnung der drei neuen Taxa (rot markiert) unter die südosteuropäischen Echinodera-Arten. UFBoot2-Werte über $90 \%$ sind an den jeweiligen Knoten angegeben.

Beschreibung und Differentialdiagnose: Größe: 2,6-3,0 mm (ohne Rüssel).

Die Unterart aus dem östlich von Sparta gelegenen Parnon-Gebirge entspricht in den äußeren Merkmalen im Wesentlichen der aus dem westlich von Sparta gelegenen Taygetos-Gebirge bekannten Art Echinodera brachati (siehe Beschreibung [WoLf, 2002]). Die wenigen Unterschiede sind jedoch augenfällig. Dies betrifft besonders die schmaleren, mindestens jedoch $2 \mathrm{x}$ längeren als breiten, etwas angehobenen Elytrenborsten, die bei E. brachati deutlich breiter sind, weniger abstehen und höchstens $1,5 \mathrm{x}$ länger als breit sind. Die 
Punktur des Pronotums ist bei der neuen Unterart feiner und unter der dichten Beschuppung kaum auszumachen, während sie bei der Unterart aus dem Taygetos viel grober, tiefer und stets deutlich zu erkennen ist. Das gilt auch für die Punktur der Elytrenstreifen, die bei E. peloponnensis unter der dichten Beschuppung kaum noch $\mathrm{zu}$ erkennen ist, während die nadelstichartige, runde Punktur in den Elytrenstreifen von E. brachati stets gut sichtbar ist. Wie diese Unterart besitzt die neue Unterart ein untersetztes Pronotum, kurzovale Elytren mit einem leichten Perlmuttglanz und einen ähnlich geformten Aedoeagus [Fig. 8, 10].

Einordnung der neuen Unterart in den Schlüssel der mediterranen, südeuropäischen Echinodera-Arten (dazu ziehe heran: STÜвEN 2018: 278):

[1] Haken am Ende der Hinterschiene bei den Männchen gewinkelt / [17'] ohne haarförmig anliegende Elytrenborsten / [18'] Elytrenborsten viel kürzer (nicht 5x-6x länger als breit wie bei E. aspromontensis) / [19`] Elytren kurzoval / [23'] Elytrenborsten kürzer, nicht mehr als 2,5x länger als breit / [28'] Elytrenborsten nicht anliegend, leicht angehoben / [30'] Pronotumpunktur gröber, nicht mit mehr als 100 feinen Punktgrübchen / [31] Pronotum vor der Basis nur schwach verrundet (bzw. annähernd parallelseitig) / [32'] Elytrenborsten länger, mindestens 1,5x länger als breit / [33] Elytrenborsten deutlich länger, mindestens $2 x$ länger als breit.

Die Unterart ist also im Echinodera-Schlüssel vor dem bekannten nominotypischen Taxon E. brachati brachati und vor E. brisouti peneckei SтÜвеN, 1998 aus Montenegro zu platzieren, die in beiden Fällen deutlich kürzere und breitere Elytrenborsten aufweisen.

Zur molekularen Einordnung der Unterart im Echinodera-Dendrogramm siehe Figur 13.

Ökologie: Der Erstautor siebte die Art in der Umgebung von Kosmas im Parnon-Gebirge aus dem Detritus von Quercus und unter Astragalus-Polstern im Schatten von Quercus-Gebüschen im August 2018 [Fig. 11].

Derivatio nominis: Die Unterart wurde nach ihrem Fundort im Süden der Peloponnes benannt.

\section{Molekulare Analyse und Einordnung der neuen Taxa im Dendrogramm der südosteuropäischen Echinodera-Arten}

Die molekulare Analyse basiert auf 23 Sequenzen von 19 südosteuropäischen Arten; inklusiv der zwei neu beschriebenen Arten und der neuen Unterart von Echinodera brachati (im Baum rot markiert). Als Außengruppe wurden zwei Sequenzen von Echinodera s. str. von den Kanarischen Inseln gewählt. Details zu den einzelnen Exemplaren, den Sammelstandorten und den jeweiligen GenBank Nummern finden sich in den Neubeschreibungen bzw. u. a. in [AsTrIN \& STÜBEN 2010] sowie [STÜBEN 2018].
Im Rahmen des Molecular Weevil Identification project (MWI, [Schütte et al. 2013] und [STÜBEN et al. 2015]) wurden 20 Sequenzen vom Zoologischen Forschungsmuseum Alexander Koenig (ZFMK, Bonn) erstellt. Die Sequenzen für die hier neu beschriebenen Arten und die Unterart lieferte das Deutsche Entomologische Institut (SDEI, Müncheberg). Belege der sequenzierten Exemplare sind beim Erstautor hinterlegt, die extrahierte DNA wird im ZFMK und SDEI aufbewahrt. Die in dieser Arbeit verwendeten Sequenzen zu den drei neuen Arten sind im Fasta-Format in Anhang 2 nochmals hinterlegt (siehe auch GenBank).

Der Ablauf der DNA Extraktion der Exemplare, die am ZMFK analysiert wurden, stimmt mit den Angaben in Schütte et al. [2013] überein. Die DNA-Extraktion am SDEI wurde mit Hilfe des E.Z.N.A. Tissue DNA Kits (Omega Bio-tek Inc., Norcross, USA), an ganzen Tieren und weitgehend nach den Angaben des Herstellers durchgeführt. Änderungen waren eine verlängerte Lyse-Zeit: statt 3 Stunden wurde über Nacht und ohne Schütteln inkubiert, und es wurde eine veränderte Menge $(2 x 100 \mu \mathrm{l})$ Elution Puffer zum Lösen der DNA von der Silikatmembran der Säule hinzugegeben. In beiden Instituten wurde ein Teilstück (658 bp) des COI Gens (Barcode Region) mit den Primern LCO1490-JJ und HCO2198-JJ [Astrin \& STÜBEN 2008] amplifiziert. Diese Primer wurden ebenfalls für die Sequenzierung benutzt. Die Sequenzen wurden manuell mit Geneious 6.1.8 [KeArse et al. 2012] überprüft und die Primersequenzen abgeschnitten. Das Alignment der Sequenzen wurde in BioEdit 7.2.5 [Hall 1999] durchgeführt.

Aus den vorhandenen Sequenzen wurde ein MaximumLikelihood-Baum zur phylogenetischen Analyse mit Hilfe der Software IQ-TREE 1.6.8 [NGUYEN et al. 2015] erstellt. Das beste Substitutionsmodell - TIM2+F+R3 - wurde durch den in IQ-TREE integrierten ModelFinder [Kalyaanamoorthy et al. 2017] bestimmt, und ein Consensus-Baum mit 1000 Ultrafast Bootstrap Replikationen [UFBoot2; HoAng et al. 2018] wurde berechnet (Fig. 13). Der Baum wurde mit Hilfe von FigTree 1.4.0 [RAMBAUt 2012] dargestellt.

\section{Danksagung}

Dankbar sind wir André Schütte und Jonas J. Astrin vom Zoologischen Forschungsmuseum Alexander Koenig (ZFMK, Bonn), die einen Teil des auf anderen Griechenland- und Italien-Exkursionen gesammelten Echinodera-Materials schon vor Jahren molekular aufbereitet haben. Unser besonderer Dank gilt Regine Stüben (Mönchengladbach), die den Erstautor auf den beiden Griechenland-Reisen begleitet hat und nicht unerheblich zur Auffindung der neuen Arten beigetragen hat. 


\section{Literatur}

Astrin, J. J. \& Stüben, P. E. 2008: Phylogeny in cryptic weevils: molecules, morphology and new genera of western Palaearctic Cryptorhynchinae (Coleoptera: Curculionidae). - Invertebrate Systematics 22: 503-522.

Astrin, J. J. \& StüBen, P. E. 2010: Molecular phylogeny of Echinodera and Ruteria (Coleoptera: Curculionidae: Cryptorhynchinae) and the parallel speciation of Canary Island weevils along replicate environmental gradients. - Invertebrate Systematics 24: 434-455.

Behne, L. 2002: Beschreibung zweier neuer RuteriaArten aus Griechenland (Mazedonien) mit einem neuen Schlüssel für die Gattung Ruteria Roudier, 1954 (Col.: Curculionidae: Cryptorhynchinae). SNUDEBILLER, Studies on taxonomy, biology and ecology of Curculionoidea 3: 226-231.

Germann, Ch.; Wolf, I. \& Schütte, A. 2015: Echinodera (Ruteria) soumasi sp. n. from Greece (Coleoptera, Curculionidae). - Mitteilungen der Schweizerischen Entomologischen Gesellschaft 88: 285-293.

HALl, T. A. 1999: BioEdit: A user-friendly biological sequence alignment editor and analysis program for Windows 95/98/NT. - Nucleic Acids Symposium Series 41: 95-98.

Hoang, D. T.; Chernomor, O.; von Haeseler, A.; Minh, B. Q. \& LE VINH, S. 2018: UFBoot2: Improving the Ultrafast Bootstrap Approximation. - Molecular Biology and Evolution 35: 518-522. - http://brownlab. mbio.ncsu.edu/JWB/papers/1999Hall1.pdf.

Kalyaanamoorthy, S.; Minh, B. Q.; Wong, T. K. F.; von Haeseler, A. \& Jermin, L. S. 2017: ModelFinder: Fast model selection for accurate phylogenetic estimates. - Nature Methods 14: 587-589.

Kearse, M.; Moir, R.; Wilson, A.; Stones-Havas, S.; Cheung, M.; Sturrock, S.; Buxton, S.; Cooper, A.; Markowitz, S.; Duran, C.; Thierer, T.; Ashton, B.; Meintjes, P. \& Drummond, A. J. 2012: Geneious Basic: an integrated and extendable desktop software platform for the organization and analysis of sequence data. - Bioinformatics 28: 1647-1649.
Nguyen, L.-T.; Schmidt, H. A.; von Haeseler, A. \& MinH, B. Q. 2015: IQ-TREE: A fast and effective stochastic algorithm for estimating maximumlikelihood phylogenies. - Molecular Biology and Evolution 32: 268-274.

Rambaut, A. 2012: FigTree (version 1.4.0). Available at. http://tree.bio.ed.ac.uk/software/figtree/.

Schütte, A.; Stüben, P. E. \& Sprick, P. 2013: The Molecular Weevil Identification Project (Coleoptera: Curculionoidea), Part I. - A contribution to Integrative Taxonomy and Phylogenetic Systematics. Snudebiller 14, no. 211: 77 pp.

StüBen, P. E. 1998: Die südeuropäischen Arten der Gattung Echinodera Wollaston und die Gattung Ruteria Roudier stat. n. (Coleoptera: Curculionidae: Cryptorhynchinae). - Beiträge zur Entomologie 48: 417-448. - https://www.contributions-to-entomology.org/article/view/1481/1480.

Stüben, P. E.; Schütte, A.; Bayer, Ch. \& Astrin, J. J. 2015: The Molecular Weevil Identification Project (Coleoptera: Curculionoidea), Part II. Towards an Integrative Taxonomy. - SNUDEBILLER: Studies on taxonomy, biology and ecology of Curculionoidea 16, no. 237: 294 pp. (Supplement, No. 238).

STÜBEN, P. E. 2018: The Cryptorhynchinae of the Western Palearctic. - Die Cryptorhynchinae der Westpaläarktis (Coleoptera: Curculionidae), Curculio Institute, Mönchengladbach: 518 pp.

Wolf, I. 2002: [new taxa]. - In: STÜBEN, P. E. \& Wolf, I.: Echinodera (s. str.) kostenbaderi n. sp. aus Norditalien und Echinodera (Dieckmannia) brachati n. sp. aus Griechenland (Curculionidae: Cryptorhynchinae). SNUDEBILLER, Studies on taxonomy, biology and ecology of Curculionoidea 3: 232-240. 


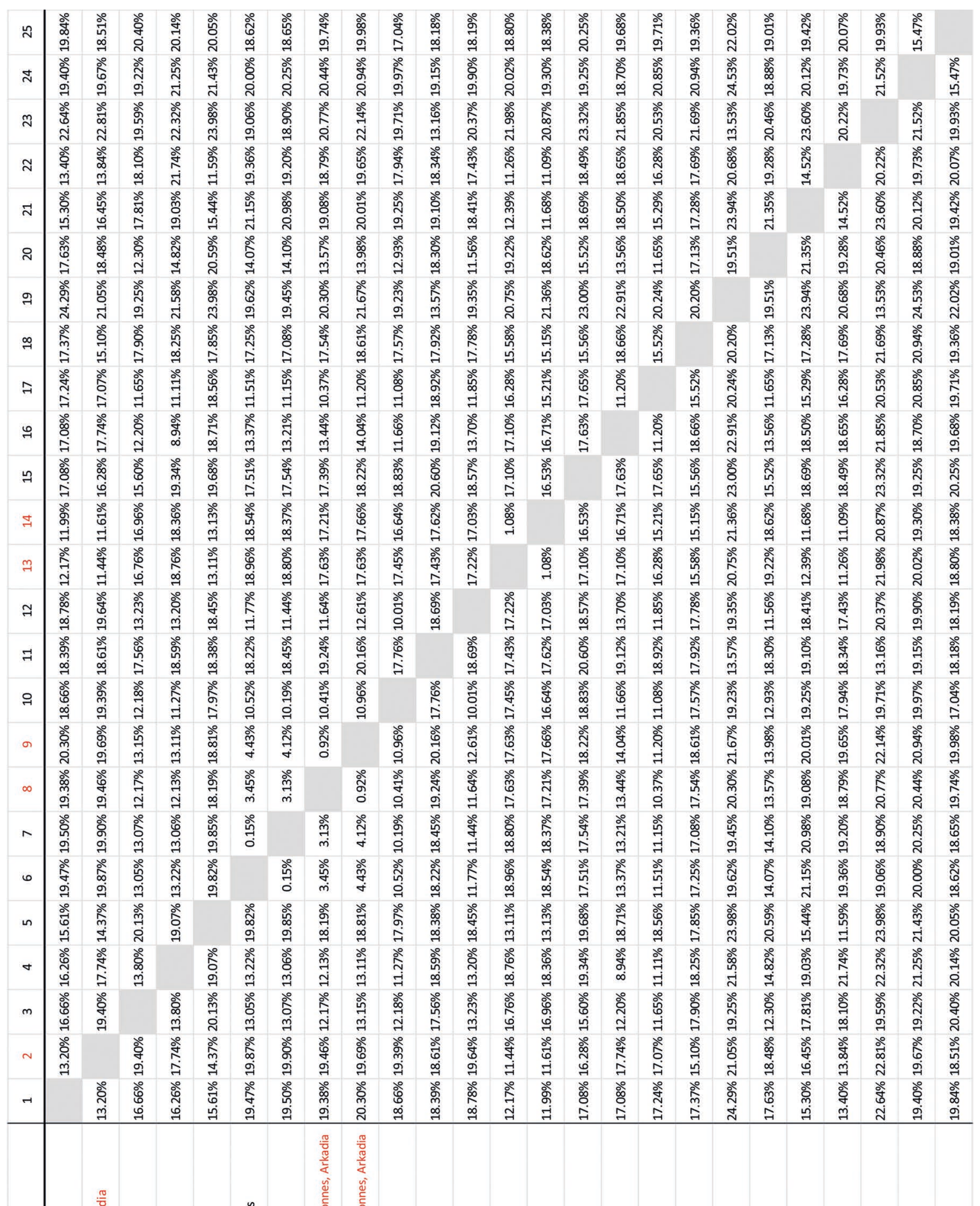




\begin{abstract}
Appendix 2
Wir sind davon überzeugt, dass molekulare Sequenzen Teil der Erstbeschreibung sein sollten, in der sie (zuerst) veröffentlicht wurden. Die Tatsache, dass sie auch separat in Genbanken hinterlegt werden, ist zweifellos eine weitere Absicherung für die langfristige Erreichbarkeit artspezifischer Barcodes. Wir stellen hier alle CO1-Sequenzdaten der drei neuen Taxa im fasta-Format zur Verfügung. Der Inhalt kann kopiert und in eine *.fasta-Textdatei eingefügt werden, die problemlos in jede Bioinformatik-Anwendung importiert werden kann.
\end{abstract}

Echinodera magnesia: Greece, (Magnesia), Pelion Mt., N Lampinou, Sammler-Nr.: 2983-PST, GenBank: MK347561 AACTTTATATTTCATTTTTGGTTCTTGATCAGGTATAGTGGGAACCTCCCTAAGTATATTAATTCGAACAGAATTAGGAAACCCAGGAACC CTAATTGGAAACGATCAAATTTACAACACAATTGTAACTGCTCATGCCTTCATTATAATTTTCTTTATAGTAATACCTGTTATGATTGGAGG ATTTGGAAATTGATTAATCCCTTTAATACTAGGGGCCCCTGATATAGCCTTCCCCCGACTAAATAATATAAGATTTTGACTACTCCCCCCAT CACTTACTCTTCTTCTAATAAGAAGAATTATTGATAAAGGCACGGGAACTGGTTGAACAGTTTATCCACCTTTATCCTCAAATATTGCCCAT GAAGGACCTTCTATTGATCTAGCCATTTTCAGGCTACATATAGCCGGAATCTCATCAATCCTAGGAGCTATAAACTTTATCTCTACAGTAAT CAATATACGCCCAATAGGTATAAAACTTGACCGAATACCTTTATTTATCTGAGCCGTAAAAATCACCGCTATTCTTCTCCTTCTATCTTTACC AGTCCTAGCAGGCGCAATTACTATACTCTTAACAGATCGTAATATTAATACATCATTTTTTGACCCTGCTGGTGGGGGAGACCCTATTCTC TATCAACATCTATTC

Echinodera magnesia: Greece, Pelion Mt. (Magnesia): 2 km W Chania, Sammler-Nr.: 3094-PST, GenBank: MK347644 AACTTTATATTTCATTTTTGGTTCTTGATCAGGTATAGTAGGAACCTCCCTAAGTATATTAATTCGAACAGAATTAGGAAACCCAGGAACC CTAATTGGAAACGATCAAATTTACAACACAATTGTAACTGCTCATGCCTTCATTATAATTTTCTTTATAGTAATACCTGTTATGATTGGAGG ATTTGGAAATTGATTAATCCCTTTAATACTAGGGGCCCCTGATATAGCCTTCCCCCGACTAAATAATATAAGATTTTGACTACTCCCCCCAT САCTTACTCTTCTTCTAATAAGAAGAATTATTGATAAAGGCACAGGAACTGGTTGAACAGTTTATCCACCTTTATCCTCAAATATTGCCCAT GAAGGACCTTCTATTGATCTAGCCATTTTCAGGCTACATATAGCCGGAATCTCATCAATCCTAGGAGCTATAAACTTTATCTCTACAGTAAT CAATATACGCCCAATAGGTATAAAACTTGACCGAATACCTTTATTTATCTGAGCCGTAAAAATCACCGCTATTCTTCTCCTTCTATCTTTACC AGTCCTAGCAGGCGCAATTACTATACTCTTAACAGATCGTAATATTAATACATCATTTTTTGACCCTGCTGGTGGGGGAGACCCTATTCTC TATCAACATCTATTC

Echinodera arcadia: Greece, Arkadia, N Tripoli, Sammler-Nr.: 3172-PST, GenBank: MK347702

AACTTTATATTTCATTTTTGGCTCATGATCAGGAATAATAGGAACTTCTCTAAGAATTCTAATTCGAGCAGAATTAGGAAACCCAGGAACA TTAATTGGAAACGATCAAATCTATAATACAATTGTGACCGCTCATGCCTTCATTATAATTTTCTTCATAGTAATACCTATCATAATTGGAGG ATTTGGAAATTGATTAATCCCCTTAATACTAGGAGCCCCTGATATAGCTTTTCCCCGTCTAAACAATATAAGATTTTGGTTACTTCCCCCAT CACTCACССTCСTTCTAATAAGAAGAATTATTGATAAAGGTGCAGGAACTGGTTGAACAGTTTACCCACCCTTATCTTCTAATATTGCCCAT GAAGGACCTTCTGTTGATCTTGCTATTTTTAGACTCCACATAGCGGGAATCTCATCAATCTTAGGAGCCATAAACTTTATTTCCACAGTAAT CAATATACGCCCAGTAGGCATAAAACTTGACCGAATACCCCTATTTATTTGAGCCGTAAAAATCACTGCTATTCTTCTCCTCCTATCTCTAC CTGTACTTGCGGGAGCAATTACTATACTCTTAACAGATCGTAATATCAATACATCATTTTTTGATCCTGCCGGAGGGGGAGACCCTATTCT CTACCAACATCTATTC

Echinodera brachati peloponensis: Greece, Arkadia, Kosmas, Sammler-Nr.: 3163-PST, GenBank: MK347695 AACTCTATATTTCATTTTTGGCTCATGGTCAGGAATAGTGGGAACATCATTAAGAATATTAATCCGAACAGAACTTGGAAGTCCTGGAACT TTAATTGGTAACGACCAAATTTATAACACAATTGTTACTGCGCATGCTTTCATTATAATCTTCTTTATAGTTATACCCATTATAATCGGAGG CTTTGGGAATTGGTTAATCCCACTAATATTAGGAGCTCCAGATATAGCTTTCCCTCGTTTAAATAATATAAGATTTTGACTACTACCTCCTT СTCTCACССTTTTACTAATAAGAAGAATTATTGATAAAGGAACAGGAACTGGTTGAACAGTTTATCCCCCTTTATCCTCCAATATTGCTCAT GAAGGAGCTTCTATTGATCTAGCCATCTTCAGCCTCCACATAGCCGGTATTTCATCAATCCTAGGAGCCATAAACTTTATTTCTACAGTAAT CAATATACGCCCAACAGGAATAAAACTAGACCGAATACCTCTATTTATTTGATCTGTAAAAATTACCGCCATTCTTCTCCTATTATCCTTAC CTGTTCTAGCAGGTGCTATCACCATACTTCTAACAGACCGAAATATTAATACATCATTTTTCGACCCAGCAGGAGGAGGAGATCCAATCCT CTACCAACATTTATTC

Echinodera brachati peloponensis: Greece, Arkadia, N Kosmas: near Moni Elonis Sammler-Nr.: 3166-PST, GenBank: MK347697 AACTCTATATTTCATTTTTGGCTCATGGTCAGGAATAGTGGGAACATCATTAAGAATGTTAATCCGAACAGAACTTGGAAGTCCTGGAAC TTTAATTGGTAACGACCAAATTTATAACACAATTGTTACTGCGCATGCTTTCATTATAATCTTCTTTATAGTTATACCCATTATAATCGGAG GCTTTGGGAATTGGTTAATCCCACTAATATTAGGAGCTCCAGATATAGCTTTCCCTCGTTTAAATAATATAAGATTTTGACTACTACCTCCT TCTCTCACССTTTTACTAATAAGAAGAATTATTGATAAAGGAACAGGAACTGGTTGAACAGTTTATCCCCCTTTATCCTCCAATATTGCTCA TGAAGGAGCTTCTATTGATCTAGCCATCTTCAGCCTCCACATAGCCGGTATTTCATCAATCCTGGGAGCCATAAACTTTATTTCTACAGTAA CCAATATACGCCCAACAGGAATAAAACTAGACCGAATACCTCTATTTATTTGATCTGTAAAAATTACCGCCATTCTTCTCCTATTATCTTTAC CTGTTCTAGCAGGTGCTATCACCATACTTCTGACAGACCGAAATATTAATACATCATTTTTCGACCCAGCAGGAGGGGGAGATCCAATCCT CTACCAACATTTATTC 